\title{
ASPECTOS SEMIÓTICOS EN EL LENGUAJE VISUAL DE LA HIBRIDACIÓN ARQUITECTÓNICA CHINO-EUROPEA
}

\author{
SEMIOTIC ASPECTS IN VISUAL LANGUAGE \\ OF THE ARCHITECTURAL CHINESE-EUROPEAN \\ HIBRIDIZATION
}

\author{
Manuel V. CASTILLA ROLDÁN \\ Universidad de Sevilla \\ mviggo@us.es
}

\begin{abstract}
Resumen: Este artículo analiza conceptos relacionados con el signo arquitectónico, y presenta una reflexión sobre aspectos semióticos del lenguaje visual de una de las obras de arte más importantes de la arquitectura en la dinastía Qing (S. XVIII): el Palacio Yuanying Guan (Vistas a un Mar distante). Se han utilizado conceptos y estructuras semióticas para interpretar la expresión de sus formas arquitectónicas y formular una comprensión posterior de dichas formas, convirtiendo cada elemento en una herramienta de comunicación.
\end{abstract}

Palabras clave: Expresión arquitectónica. Semiótica. Perspectiva lineal. Yuanying Guan.

Abstract: This article analyses some concepts related to the architectural sign and presents itself a semiotic reading of one of the most important works of art of the architecture in Qing dynasty (18 ${ }^{\text {th }}$ Century): the 
Yuanying Guan (View of the Distant Sea) pavilion. Semiotic structures were employed to interpret the expression of architectural forms and formulate a subsequent understanding of these forms by turning each element into a communication tool.

Key Words: Architectural expression. Semiotic. Linear perspective. Yuanying Guan.

\section{INTRODUCCIÓN}

La Semiótica se ha definido como la ciencia de los signos y analiza cualquier sistema como un conjunto de signos. En este campo, han sido fundamentales las contribuciones del lingüista suizo Ferdinand de Saussure (1857-1913) y el filósofo americano, Charles Sanders Peirce (1839-1914). Ellos, iniciaron la aplicación de la Semiótica al lenguaje, y elevaron su estatus a la categoría de ciencia. Posteriormente, Roland Barthes (1915-1980) y Umberto Eco (1932-2016), extendieron las teorías semióticas de Peirce a diferentes tipos de mensajes, y entre ellos, a los que están relacionados con el proceso del diseño arquitectónico (JuodinytéKuznetsova, 2011: 1269-1280).

Históricamente, en el significado y expresión arquitectónica han intervenido un conjunto de factores de tipo religioso, artístico, cultural, social, o político, así como otros elementos, en forma de signos que transmiten distintos significados. Sin embargo, existe un común denominador en la concepción de un texto arquitectónico: el espacio y su contexto. Para K. Juodinyté-Kuznetsova (2011: 1271), "la arquitectura es un tipo específico de semiótica espacial”. Pero no debemos limitarnos a considerar la dimensión espacial y el contexto como únicos atributos de la arquitectura. Existen otras dimensiones: tectónica, pragmática, simbólica, entre otras, que deben tenerse en cuenta en la concepción del lenguaje visual de sus distintas manifestaciones (Paniagua, 2013: 526). 
Analizaremos en qué medida la Semiótica puede explorar la complejidad del discurso visual del conjunto arquitectónico del Yuanying Guan (Vistas a un Mar Distante), presentando aspectos semióticos del lenguaje visual que la explique claramente. Esta línea de conocimiento se ha mantenido relativamente desconocida en la arquitectura actual, entre otros motivos, porque algunos investigadores modernos han sugerido que la arquitectura no puede ser reconocida fuera de su entorno universal. Según Taurens, "the architectural meaning is understood as context-dependent, the term 'context' being under-stood not only as a spatial context of a greater architectural expression, but more widely” (Taurens, 2008: 77).

Partiendo de la base de que la concepción arquitectónica es un lenguaje de comunicación visual mediante un sistema de signos, sin olvidar su funcionalidad, en ella, no puede existir un significante que no tenga significado. En un sistema de escritura arquitectónica aceptable, muchas propiedades lingüísticas de los proyectos arquitectónicos son fáciles de reconocer y se pueden interpretar durante la construcción, o en las distintas fases del diseño arquitectónico. En este caso, la lectura de la arquitectura tiene una fácil comprensión cuando las condiciones textuales son evidentes. Entre los lenguajes evidentes podemos citar, el uso al que se destina un edificio, la tecnología empleada, materiales utilizados, su forma constructiva y su organización espacial (dimensión tectónica). Pero existe una lectura difícil de la expresión arquitectónica cuando la propia arquitectura da lugar a significados que son legibles a través de un análisis complejo. Las condiciones textuales menos evidentes pertenecen al campo de la subjetividad y de la interpretación. Jencks justifica estos inconvenientes cuando dice que, "las palabras arquitectónicas son más elásticas y polimorfas que su variedad escrita y hablada" (Jencks, 1984: 104).

Por ello, leer la arquitectura, no implica la existencia de un lenguaje textual lineal, por lo que dicha lectura no está controlada por la intencionalidad de los arquitectos y diseñadores, de la misma forma que un pintor no puede predecir, cómo interpretará su pintura un observador 
determinado. El lenguaje visual arquitectónico necesita organizar los distintos mensajes y signos de acuerdo con ciertas convenciones que los semióticos llaman códigos. Estas convenciones representan en Semiótica la dimensión sociocultural. Los signos serán interpretados por la audiencia en base a las reglas y convenios de su cultura y determinan cómo y en qué contextos se utilizan y se combinan para expresar mensajes más complejos (Fiske, 1990: 19). En nuestro caso, la amplitud y profundidad científicotécnica de la hibridación arquitectónica chino-europea durante el reinado del emperador Qianlong (1735-1796) y algunos conceptos de la filosofía taoísta podrían utilizarse como códigos suficientes para fundamentar los aspectos semióticos del lenguaje visual del palacio View of the Distant Sea (Yuanying Guan). En este sentido, la hibridación pictórica y arquitectónica chino-europea en los comienzos de la dinastía Qing conjugó la concepción cosmológica de los emperadores en la construcción de grandes jardines y palacios, con los intereses apostólicos de los artistas jesuitas. En primer lugar, estos utilizaron un "lenguaje arquitectónico" basado en la perspectiva lineal europea para expresar en muchos casos su intencionalidad apostólica (Castilla, 2015). En segundo lugar, mantuvieron las tradiciones chinas para construir un conjunto de jardines y palacios de estilo europeo como texto simbólico, dibujando un espacio paradisíaco para satisfacción del emperador.

\section{FUNDAMENTOS TEÓRICOS}

\subsection{Antecedentes}

Prácticamente de forma simultánea, Ferdinand de Saussure (18571913) y Charles Sanders Peirce (1838-1914), anunciaron sendas teorías sobre los signos. Para de Saussure, la "lengua es un sistema de signos que expresan ideas, y, por lo tanto, comparable a la escritura". Para él, "es posible concebir una ciencia que estudie la vida de los signos en el seno de la vida social, y esta ciencia nos enseñará en qué consisten los signos y 
qué leyes los rigen". Saussure definió un signo diádico con dos elementos: significante (imagen de un signo) y significado (concepto de la imagen). Ambos son imprescindibles en el modelo de Saussure. Su concepción del significado dentro del sistema es puramente estructural, dando primacía a las relaciones sobre todo lo demás (estructuralismo). "Signs refer primarily to each other. Within the language system, everything depends on relations" (Saussure, 1983: 121).

Para Charles Sanders Peirce, la Semiótica se convierte en disciplina y ofrece tres modos de signos que tienen diferentes formas de comunicar del arte visual: icónico, simbólico e indéxico (Chandler, 2007: 40-41). Peirce define el signo como un modelo triádico, con tres elementos: representamen o significante (forma que toma el signo), interpretant o significado (sentido que se da al signo) y object (objeto al cual se refiere el signo). En la teoría peirceana, el proceso de interpretación puede continuar hasta el infinito (Chandler, 2007: 32-33). Para Martin, la "semiótica explora el significado mediante el descubrimiento de las capas más profundas de un texto" (Martin y Ringham, 2000: 118).

Básicamente, entre 1970-90 se publicaron diferentes trabajos que han ayudado a la interpretación de las formas arquitectónicas desde el punto de vista semiótico. En 1969, Charles Jencks y George Baird publicaron una colección de ensayos titulada Meaning and Architecture de gran interés desde un punto de vista semántico. Sobre esta colección J. A. Hale escribe:

One of the first manifestations of this semantic tendency in architecture was the collection of essays published in 1969, entitled Meaning and Architecture and edited by Charles Jencks and George Baird. Jencks went on to champion the use of semantic references in buildings, which became the basis of what we now refer to as the "language" of postmodernism in architecture (Hale, 2000: 146-147). 
A principios de 1970, se publicó un interesante trabajo de Aldo Rossi, titulado The Architecture of the City. En 1977, Geoffrey Broadbent explora las relaciones entre forma y significado en un ensayo titulado “A Plain Man's Guide to the Theory of Signs in Architecture". En 1985 la obra de Nelson Goodman titulada How Buildings Mean y el trabajo The Language of Architecture de Martin Donougho, constituyeron serios intentos de utilización de la Semiótica como herramienta ideal para comprender la expresión de las formas arquitectónicas. En 1982, A. J. Greimas y J. Courtés publican un diccionario conceptual, Semiotics and Language: An analytical Dictionary, o diccionario razonado de la teoría del lenguaje. En 1995 (Agrest and Gandelson, 1995: 41-50) publican un libro titulado Semiotics and Architecture, en el que clarifican que la Arquitectura no tiene un significado interno, sino que su significado está relacionado con la sociedad y la cultura. En 1997, U. Eco publica Function and Sign: the Semiotics of Architecture que junto a los trabajos desarrollados por Algirdas Julien Greimás y la Escuela de París consideran que el diseño arquitectónico es el resultado de un proceso sociocultural. A partir del año 2000, aparecen algunos textos que exponen las bases de la Semiótica con gran claridad, Semiotics: the Basis (Chandler, 2007), y otros trabajos directamente relacionados con líneas de investigación en el ámbito del análisis semiótico integrado en la Arquitectura. Entre ellos son dignos de mención, Meaning and context in the lenguaje of Architecture (Taurens, 2008), Semiotics of Architectural Graphics (Tasheva, 2012), o La Arquitectura y su significación pragmática y tectónica (Paniagua, 2013), que sugieren que el principal sistema expresivo que da un nuevo significado al diseño arquitectónico es el sistema semiótico o sistema de los signos y analizan las características de la significación arquitectónica.

Pero si los fenómenos culturales principalmente son sistemas de signos, la cultura esencialmente es comunicación. Por ello, debido a la índole de la realidad que la arquitectura quiere captar, esta es un campo en el que la Semiótica encuentra determinadas dificultades. Los objetos arquitectónicos como actos de comunicación no deben excluir su 
funcionalidad. "En este sentido, lo que me permite el uso de la arquitectura no solamente son las funciones posibles, sino, sobre todo, los significados vinculados a ellas, que me predisponen para el uso funcional" (Eco, 1986: 252-256). Sin embargo, los escasos trabajos existentes relacionados con la hibridación arquitectónica chino-europea del siglo XVIII y sus significados, están basados en lo puramente estético. Entre ellos debemos destacar China and Europe intertwined: a new view of the European Sector of the Chang Chun Yuan, de Victoria M. Siu (2012), que ofrece una visión detallista de algunos símbolos de los Palacios Europeos. En esta misma línea podemos situar A Jesuit Garden in Beijing and Early Modern Chinese Culture, de Hui Zou (2011) y Stanging Europe: Theatricality and Painting at the Chinese Imperial Court, de Kristina Kleutghen (2013).

\subsection{Semiótica: método para la lectura de un texto arquitectónico}

Una visión de la naturaleza lingüística de las formas arquitectónicas puede explicarse debido al cambio de significados que ocurre en los edificios y sus partes constituyentes a través del tiempo (Eco, 1986: 271-273). Un ejemplo de ello es el Partenón, cuyo origen fue un lugar para el culto y hoy es un símbolo de la cultura griega. Es evidente que los monumentos históricos permanecen en el tiempo, pero no se puede asegurar que su significación se conserve, por lo que estilos y formas arquitectónicas pueden desaparecer. Un mensaje decodificado con facilidad por un observador del siglo XVIII posiblemente sea inaccesible a un observador contemporáneo. En este sentido, Magariños en La Semiótica de los bordes escribe:

[...] lo que implica entender el significado como un efecto históricamente situado y no como una sustancia esencial y universal. Según esto, la primera tarea de la Semiótica, desde el punto de vista lógico, consiste en explicar, no ya el significado de los fenómenos sociales, sino, antes que nada, el proceso de 
producción, interpretación y transformación de tal o de tales significados. [...] Porque el significado [...] es un estado de tránsito, que se hizo posible por haber existido un antes, desde el cual el significado que en él tenía ese fenómeno, contenía también, en sus bordes, la posibilidad de que se formulara el que tiene ahora (Magariños, 2007: 99-100).

Basándonos en la metodología descrita en Amirmasoud (2015: 2460-2462), para la lectura de un texto arquitectónico utilizaremos un conjunto de descriptores relevantes que definen determinadas capas o modelos de significación: histórico, estético, simbólico, funcional, económico, etc., que debidamente interrelacionados forman un texto arquitectónico. La lectura de este texto tiene como objetivo reproducir un objeto arquitectónico en función de aquellas interrelaciones y la propia interpretación del receptor del objeto. En este artículo, el sistema semiótico generado del caso de estudio propuesto (texto arquitectónico), se ha desarrollado a partir de las siguientes consideraciones:

$\square \quad$ Un sistema de capas y subcapas transmite un mensaje o un conjunto de mensajes que a su vez se transfieren al destinatario. Los diferentes códigos contenidos en el texto se transfieren a través de su significación y relaciones intertextuales. El destinatario intentará decodificar y transmitir el significado del texto, sus diferentes modelos de significación, sus relaciones y su propio punto de vista.

$\square \quad$ Para comprender, interpretar los diferentes mensajes, el destinatario debe utilizar una disciplina coherente que reconozca los elementos del texto e interprete el significado. Esta disciplina es la Semiótica, o ciencia de los signos, que analiza cualquier texto, como un "sistema de signos" mediante tres funciones debidamente interrelacionadas: el significado de los signos (semántica), la relación entre ellos 
(sintáctica), y la lectura que hace el destinatario (pragmática) (Nöth, 1990: 49-51). Los signos no tienen significados de forma aislada, sino que obtienen su significado cuando se insertan en un texto asociado a textos anteriores y funcionan como introducción a otros textos. A la relación entre las tres funciones, Eco le llama sistema de proceso.

$\square \quad$ Finalmente, la Tabla 1 resume la interpretación del sistema semiótico generado con la ayuda de conceptos y herramientas semióticas. Éstas han sido utilizadas en el análisis semiótico del Yuanying Guan para facilitar la lectura de su lenguaje arquitectónico. Entre ellas citaremos: los Modos de signos, Código, Denotación, Connotación, Paradigma, Sintagma, Metáfora, Metonimia y Articulación.

En consecuencia, la arquitectura puede ser concebida como un texto espacial con múltiples connotaciones dentro de sus propios códigos. Si nos apoyamos en la frase de Charles S. Peirce, "we think only in signs" (Peirce, 1931-1958: 2.302), deduciremos que la Semiótica, puede dar respuestas a la lectura de una arquitectura de signos. Si, además, los fenómenos culturales son sistemas de signos, entonces:

[...] la cultura esencialmente es un proceso de comunicación, y uno de los sectores en el que la Semiótica encuentra mayores dificultades, por la indole de la realidad que pretende captar, es el de la arquitectura. [....] El examen fenomenológico de nuestras relaciones con el objeto arquitectónico ya nos indica que por lo general disfrutamos de la arquitectura como acto de comunicación, sin excluir su funcionalidad (Eco, 1986: 252256).

Eminentes post-estructuralistas como Barthes y Derrida investigaron el texto en relación con otros textos fácilmente reconocibles y 
propusieron un sistema de capas en la lectura del texto. Barthes considera el texto como un producto de elementos sociales, históricos y culturales específicos, por lo que en su esencia interviene principalmente el contexto y sus relaciones intertextuales. Para Eco, el modelo funcional es prioritario sobre todos los demás. La estructura del texto es multidimensional y genera un significado según el contexto: "Textual reading does not look for discovering the reality and meaning, however, it produces a semiotic structure" (Barthes, 1972: 55). Debemos dejar bien claro, como dice Eco, que en este trabajo "nos hemos de ocupar de la manera de leer un objeto arquitectónico y no de la manera de leer un proyecto" (Eco, 1986: 281).

\section{ASPECTOS SEMIÓTICOS EN EL LENGUAJE VISUAL DEL YUANYING GUAN}

\subsection{Antecedentes históricos}

El apogeo de la dinastía Qing (1644-1911) coincidió con los reinados de los emperadores Kangxi (reinó entre 1662-1722), Yongzheng (reinó entre 1723-1735) y Qianlong (reinó entre 1736-1795) (Rawski y Rawson, 2006), en los que China creció y vivió en los aspectos científicos y artísticos un periodo de gran prosperidad y esplendor. El largo reinado de este último proporcionó una etapa de estabilidad después de la caótica transición dinástica y disfrutó de un periodo de gran motivación por las actividades, culturales y artísticas, siendo la pintura y la arquitectura, junto a la astronomía y cartografía, las que tuvieron un intenso y original desarrollo. Durante la dinastía Ming y principios de la Qing, la Compañía de Jesús culturalmente polifacética, abanderó la enseñanza de la religión e impulsó las ciencias y las artes durante su acción misional (Standaert, 2007: 39). Los jesuitas aprovecharon la capacidad expresiva del Renacimiento y del Barroco para ejercer en la corte imperial como verdaderos artistas en la utilización de la perspectiva lineal occidental. Desde el punto de vista pictórico-arquitectónico, la llegada del jesuita Giuseppe Castiglione 
(nombre chino, Lang Shining, 1688-1766), que provenía de la tradición barroca, significó un referente en la pintura y arquitectura ilusionista y el diseño arquitectónico en Asia Oriental (Castilla, 2015, 2016).

\subsection{Lenguaje visual en la arquitectura del Yuanying Guan (Vistas a un Mar Distante)}

En primer lugar, en la hibridación arquitectónica chino-europea, los artistas jesuitas utilizaron un lenguaje arquitectónico basado en la adecuación de la perspectiva lineal europea o xianfa ${ }^{1}$. Esta técnica estaba directamente relacionada con la cultura occidental y con el concepto chino de distancia profunda ${ }^{2}$, donde la visualización de una imagen se hacía a través de los sentidos y sobre todo de la vista. Al simbolismo y sensibilidad de la mentalidad asiática, Castiglione añadió el misterio del ilusionismo mágico que encierra la perspectiva matemática lineal europea. Este método conectó la religiosidad de los jesuitas con la cosmología china, ya que para los chinos el punto focal (punto de fuga o punto del infinito) se relacionó con el origen del mundo (Zou, 2005: 193), en el más estricto sentido taoísta y para los jesuitas el punto de fuga conducía hacia la gloria de Dios ${ }^{3}$.

En segundo lugar, mantuvieron las tradiciones chinas para construir un conjunto de palacios de estilo europeo al noreste de los Jardines Yuanming Yuan ${ }^{4}$. Estos jardines fueron construidos en 1709 por

\footnotetext{
${ }^{1}$ Véase Elisabetta Corsi (2004), La fábrica de las ilusiones, Los jesuitas y la difusión de la perspectiva lineal en China (1698-1766); además la reseña de Flora Botton, en Estudios de Asia y África 40.2 (127), 465-467.

${ }^{2}$ Fue una de las tres distancias propuestas por Guo Xi para la pintura china. La distancia profunda, se definió como aquella que sobrepasa el espacio físico y se adentra en el espacio espiritual que constituye la propia esencia de la naturaleza.

${ }^{3}$ Andrea Pozzo (1741-1758), Perspectiva Pictorum et Arquitectorum/ Prospectiva De'Pittori e Architecti D’Andrea Pozzo della Compagnia di Gesú, Doble texto latín/ italiano publicado en Roma, vol. I (1741), vol. II (1758). Ver la edición de 1989.

${ }^{4}$ H. Zou (2011: 15), en A Jesuit Garden in Beijing and Early Modern Chinese Culture, señala: "In modern-day China, when people hear the term yuanming (literally, round brightness), they probably think of two wonders: one is the bright full moon appearing at the middle of each month; another is the Yuanming Yuan, literally, Garden of Round
} 
el emperador Yongzheng y posteriormente ampliados, utilizándolos como residencia permanente y lugar de audiencias. Por sus enormes dimensiones y artificialidad de sus paisajes, frente a la naturalidad de los jardines chinos, fue considerado un jardín insólito.

Qianlong, que había conocido grandes obras sobre arquitectura europea, tuvo la revelación y la idea (Beurdeley, 1997: 124) de construir en los jardines del Yuanming Yuan un conjunto de palacios de estilo europeo y originales fuentes. La gran empresa fue encomendada en 1747 al jesuita Giuseppe Castiglione (Pirazzoli-T'Serstevens, 2007: 134), que diseñó distintos pabellones de estilo occidental en un área en forma de $\mathrm{T}$, situada al noreste del Jardín de la Perfecta Brillantez (nombre jesuítico) o Yuanming Yuan (nombre chino).

Los diseños efectuados por Castiglione estuvieron realizados en un original barroco, donde algunos elementos ornamentales y arquitectónicos, en cierta forma, recordaban las arquitecturas de Guarino Guarini y Borromini (Beurdeley, 1971: 45-67). La escenografía de este Jardín Europeo se identificó con la miniaturización de un reino mágico asociado al universo, la inmortalidad y el poder. La forma rectangular del encaje del Jardín Europeo dentro del Yuanming Yuan delimitaba con perfección las fronteras de la perfecta brillantez, como expresó el emperador Qianlong: "[...] encircle the cosmos and things into the round brightness". Esta denominación implica, perfección y excelencia (Wong, 2001: 1). Particularmente, un mensaje que trasmite el Jardín Europeo es la delimitación de la brillantez, expresado en la nota 12. Este rodeo de la redonda brillantez se obtuvo geometrizando las fronteras de los Palacios Europeos que, por otra parte, eran los más alejados dentro del Paraíso Perdido, como llama Young-Tsu Wong a los jardines Yuanming Yuan

Brightness, which exists only in their minds".

${ }^{5}$ Qianlong, en su "Later Record of the Garden of Round Brightness" escribió: “[...] surrounded all things and thus came to form the round brightness". La cita está tomada de la tesis doctoral de Hui Zou, traductor del documento original. Según Zou, la frase en cursiva significa literalmente: "[...] encircle the cosmos all things and thus came to form into round brightness". 
(Wong, 2001).

El monumental proyecto fue considerado como uno de los mayores exponentes de la hibridación pictórico-arquitectónica chino-europea del siglo XVIII ${ }^{6}$ (Tabla 1. Indicador A). El objetivo principal fue crear un espacio, donde el emperador pudiera visualizar el mundo como un microcosmos escénico de jardines, lagos, fuentes y palacios, y a su vez, que los artistas jesuitas pudieran ejercer su misión apostólica. Esta sucesión de escenas demostró, no solo la asociación de "montañas y aguas" de los jardines chinos, sino que también definió la dimensión sensorial de la filosofía y religión china. Es cierto que existió un significado material en las formas arquitectónicas del Yuanming Yuan, pero no podemos olvidar su impacto en los sentidos. Por ello, el significado de redonda brillantez (versión china), no solo debe interpretarse como una luna llena o brillantez perfecta (versión jesuítica), sino también, como un signo capaz de activar una dimensión sensorial. En nuestro caso, la integración escénica en el Complejo del Yuanying Guan ${ }^{7}$ (Tabla 1. Indicador B), no solo se referirá a

${ }^{6}$ En la dinastía Qing, el llamado Canton System, juega un papel fundamental en la popularización de construcciones comerciales y residenciales de estilo occidental en China. Durante el reinado del emperador Qianlong, periodo de gran prosperidad económica, este encargó al pintor, arquitecto y misionero jesuita, hermano Lego Giuseppe Castiglione la responsabilidad de dirigir al equipo que diseñó dichos Palacios o Western Mansions (Jardín Europeo). La creación de G. Castiglione estuvo guiada, en primer lugar, por el uso de la perspectiva lineal renacentista y en segundo lugar, y dado que el jardín y la monumentalidad, formaban parte de la cultura tradicional china, por el intento de crear un jardín geometrizado (significante) con claras influencias chinas y europeas, para deleite del emperador. El empleo de la Óptica Geométrica diferenció el llamado Jardín Europeo del resto del Yuanming Yuan, "sinuoso y serpenteante". La delimitación de la brillantez es el significado de la geometrización. El simbolismo del signo está basado en su convencionalidad: rodear. Su disposición sintagmática representa un "todo" cuyo significado es el Jardín Europeo que denota un lugar determinado y connota con las funciones, pabellones, jardines etc. de este lugar. Rodear la perfecta brillantez es un signo metafórico evidente. Existe también una articulación que determina las reglas para combinar paradigmas dentro del jardín (Tabla 1. Indicador A).

${ }^{7}$ El palacio Yuanying Guan tiene un significado global como mirador de escenas paisajísticas y centro de recepción de visitantes y embajadas. Su modo de signo indéxico le conecta de forma existencial a su significado de auténtico "belvedere". Entre los tres palacios que utilizan el término guan (mirador), el Yuanying Guan es un icono porque 
sus monumentales construcciones y sus simbólicas interpretaciones, sino también a la activación mental con conexiones culturales y artísticas como la poesía o la pintura.

En estos recintos palaciegos, los elementos constructivos y decorativos que implicaban la presencia de la estética china en el paisaje, la vegetación (elemento fundamental del jardín por su simbolismo), la cosmología, el trono como elemento de poder, la roca o el agua, junto a otros propios de la cultura occidental, como la perspectiva lineal renacentista. Estos elementos constituyeron las fuentes más frecuentes de inspiración en la obra arquitectónica que los artistas jesuitas desarrollaron durante su acción misional en Asia.

La Figura 1 representa un plano de situación del Jardín Europeo y la Figura 2 un plano del Jardín Europeo con la ubicación de cada uno de los recintos que ejemplifican la mutua colaboración entre Europa y China, en términos de técnicas constructivas y estilos decorativos (Waley-Cohen, 1999: 116):

posee ese carácter claramente definido. Respecto del Complejo (sintagma), este palacio es un paradigma porque todos sus signos están asociados para definir su estructura mediante los códigos adecuados. Además, denota su propio significado: está construido sobre una plataforma elevada y su orientación le permite recibir la luz del Sur. Connota directamente del significado hacia vistas de paisajes, lagos y jardines, iluminación, Sur. Desde el punto de vista metafórico, su propio nombre (View of the Distant Sea o Vistas a un Mar Distante) es una bella metáfora. Sin embargo, la parte del título Sea=Mar invoca a todos los lagos visibles desde el trono del Palacio. En este sentido el signo es metonímico. Su doble articulación permite un análisis del significante en dos niveles. En un primer nivel hay que considerar el conjunto de unidades significativas con significante y significado: geometría, planimetría, decoración, ventanas, etc. Estos signos a su vez están compuestos por elementos de un nivel inferior de articulación (segundo nivel) (Tabla 1. Indicador B). 


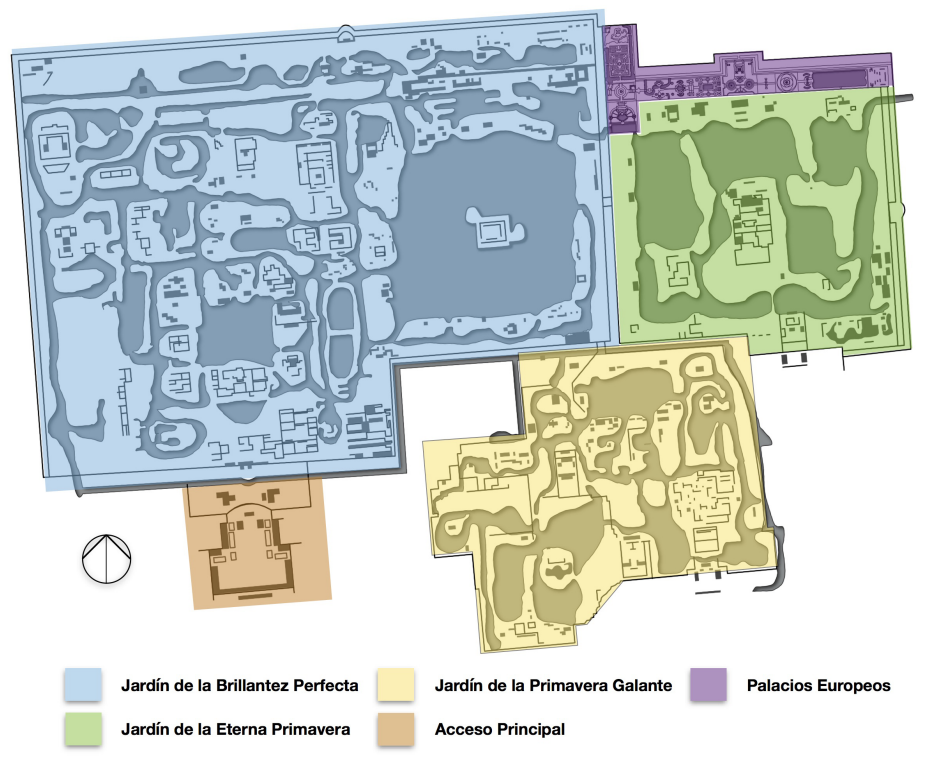

Figura 1. Situación y emplazamiento de los jardines y Palacios Europeos.

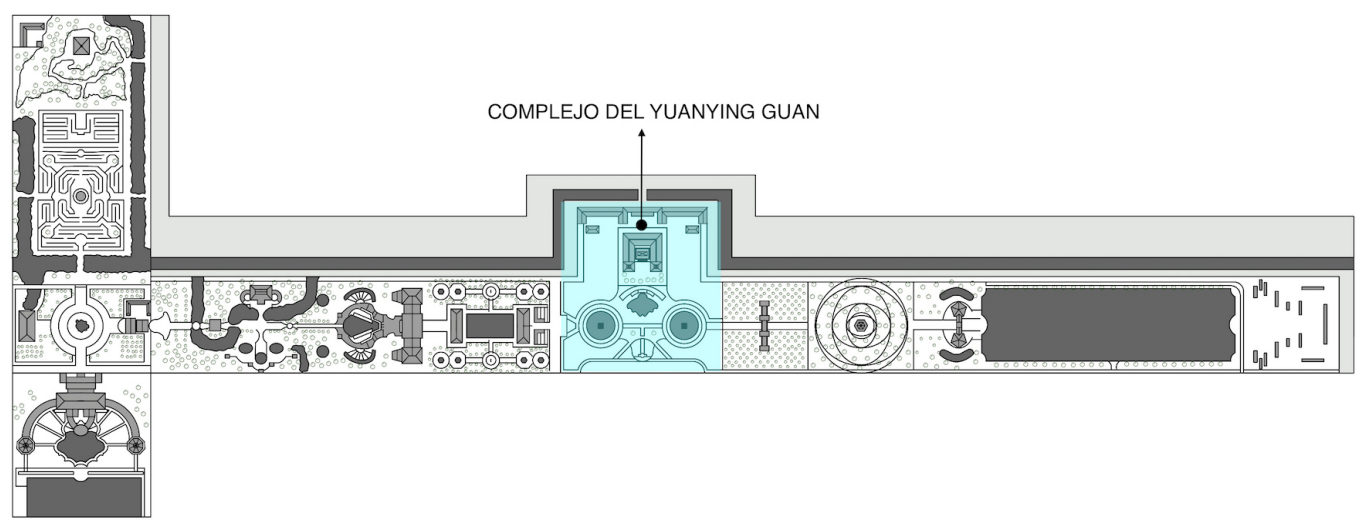

Figura 2. Plano de los Palacios Europeos. Reconstrucción del autor. 
Una lectura semiótica del interior y exterior de los Palacios Europeos nos llevaría a una representación escénica de extraordinarias dimensiones. Sin embargo, dichos palacios fueron salvajemente saqueados y destruidos por tropas anglo-francesas en $1860^{8}$ (Barmé, 1996: 126-137), quedando de ellos 20 grabados realizados por Yi Lantai (1749-1789), pintor de la corte, por encargo del Emperador Qianlong ${ }^{9}$ el año 1783 (Thiriez, 1994: 12).

La totalidad del complejo del Yuanying Guan se compone de tres paradigmas: el Palacio Vistas a un Mar Distante (Yuanying Guan propiamente dicho), la Gran Fuente (Dashuifa) y el Trono (Throne). Para leer los mensajes incorporados por Yuanying Guan debe tenerse en cuenta que solo disponemos de 3 grabados (Figura 3, Figura 4 y Figura 5) de los 20 encargados por el emperador Qianlong y la escasa información existente en los archivos imperiales, bibliografía y en los restos de su destrucción. Con estos datos, el análisis semiótico únicamente podremos dirigirlo utilizando herramientas y estructuras conceptuales. Por lo tanto, la lectura se referirá sustancialmente a la originalidad del mensaje visual y estético de dicho objeto arquitectónico y su significado en su propio contexto. Nos referiremos a las relaciones jerárquicas de los signos

\footnotetext{
${ }^{8} \mathrm{Su}$ destrucción estuvo directamente relacionada con la segunda Guerra del Opio, que estalló en 1856, y en la que los franceses, con Napoleón III, se unieron a los ingleses para derrotar al imperio chino.

${ }^{9}$ Terminada la construcción de los Palacios Europeos, Qianlong encargó a Yi Lantai (17491789), alumno aventajado de Castiglione, la creación de 20 grabados en perspectiva para la obra Pictures of the European Palaces and Waterworks. Estos combinan la perspectiva y el ilusionismo pictórico europeos, con la teoría y práctica del diseño de los jardines chinos, y representan una recreación realista hasta entonces desconocida. La manuscrita se encuentra en la Biblioteca Nacional de Francia, París, donde se existe además otra versión grabada. Existen otras series completas en la Biblioteca Pública de Nueva York y en Évora (Portugal), una serie que no está probado que sea original. Existe además una serie de los grabados de los Palacios Europeos en la Biblioteca de la Universidad de Manchester con la particularidad de que consta de 21 láminas, una de ellas a color, desconociéndose por el momento su autoría e intencionalidad. Otras series, algunas de ellas incompletas, en el Museo Británico y Museo Victoria \& Albert. En la actualidad, existen unas representaciones halladas en Yuxian (China) de dudosa relación con las "Western Mansions" del Yuanming Yuan, aunque están en proceso de análisis.
} 
externos que transmiten los grabados, no pudiéndose hacer una lectura completa del proyecto ni de la posible riqueza de su decoración interior (Pirazzoli-T'Serstevens, 2007: 144).

Nuestro objetivo es llegar a comprender esta dimensión simbólica mediante el lenguaje visual de la expresión arquitectónica del palacio, teniendo en cuenta que la gramática del diseño empleada por G. Castiglione fue una híbrida interpretación chino-europea escénica del jardín. Su trinomio inculturador, ciencia-arte-religión, siempre estuvo fundamentado en una base científica, un propósito artístico y una finalidad evangelizadora. Esta convergencia de intereses no es ajena al principal objetivo del misionero jesuita: conectar su obra con la presencia de Dios como fuente de realidad geométrica a través de sus pinturas y proyectos arquitectónicos. En este sentido, Castiglione, no solo respetó la tradición china, sino que también sintió la necesidad de revelar su propia inspiración y sus creencias religiosas. Según Loerh, el arquitecto jesuita fue fiel al simbolismo chino, pero también es posible creer que los artistas misioneros planearon deliberadamente aquellas características que agradaban al emperador y, a la vez, creaban una oportunidad de acción apostólica (Loehr, 1940: 96-98).

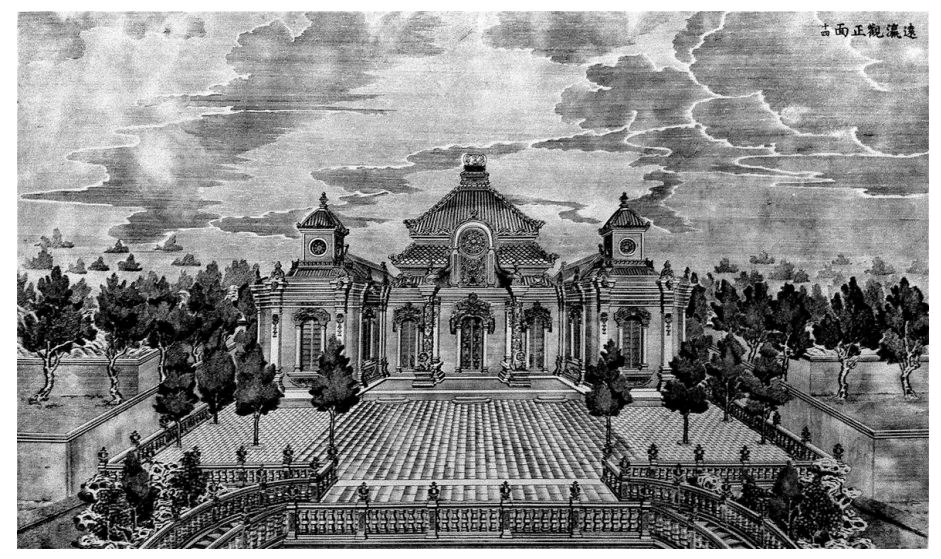

Figura 3. Yuanying Guan (Vistas a un Mar Distante). Yi Lantai (17491789). Grabado $n^{\circ} 14$. Bibliothèque Nationale de France, Paris. 


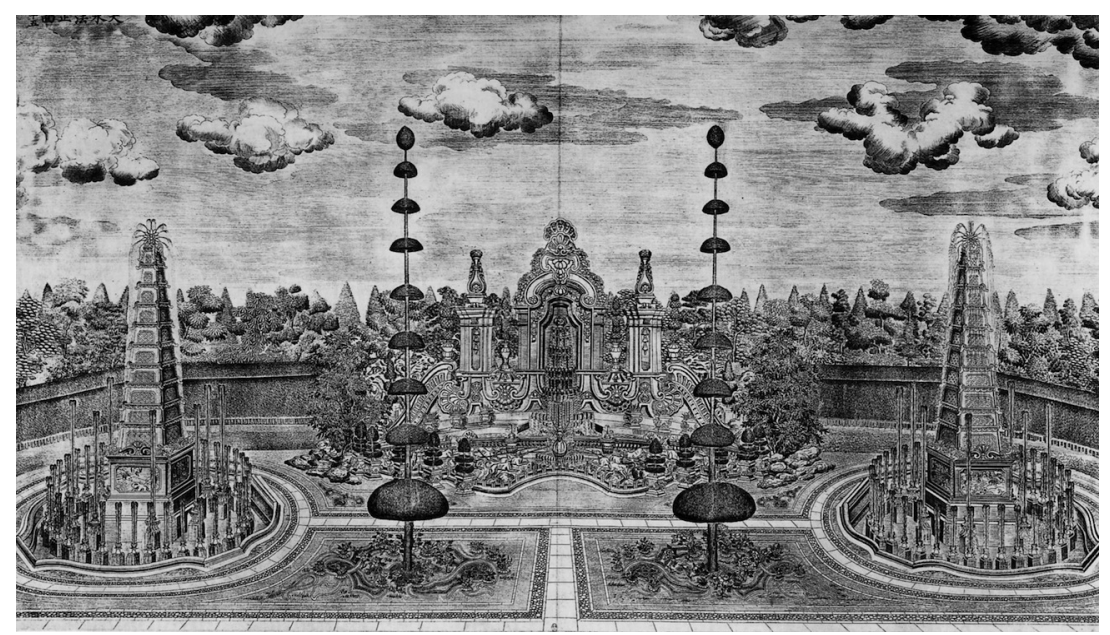

Figura 4. Dashuifa. (Great Fountain). Yi Lantai (1749-1789). Grabado ${ }^{\circ}$ 15. Bibliothèque Nationale de France, Paris.

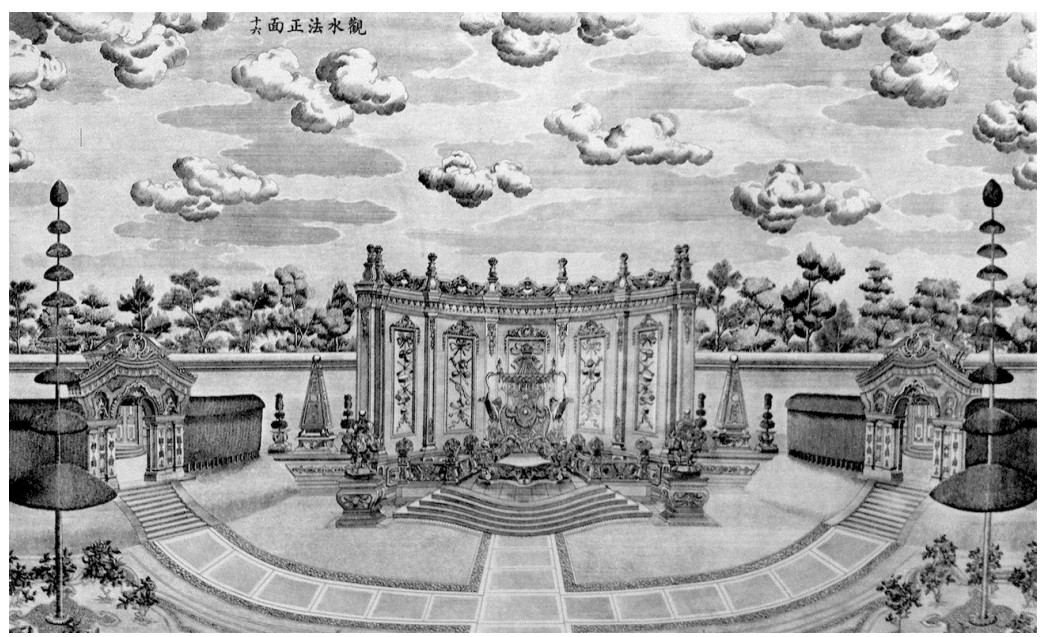

Figura 5. Throne. Vista frontal. Yi Lantai (1749-1789), Grabado n ${ }^{\circ} 16$ Bibliothèque Nationale de France, Paris. 
Castiglione diseñó espectaculares escenarios utilizando para ello los códigos de la perspectiva lineal renacentista, consiguiendo puntos de fuga óptimos para cualquier observador. De estos escenarios emergían un conjunto de signos codificados por la ortogonalidad de los ejes y la simetría bilateral, cuya estrategia fue ampliamente utilizada por el jesuita (Figura 6):

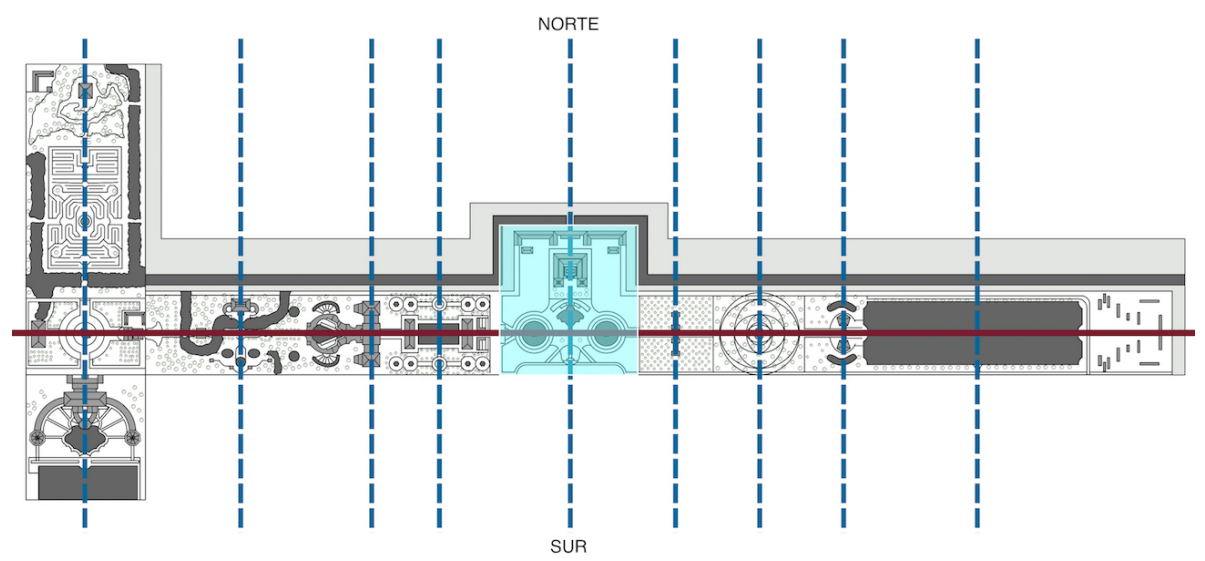

Figura 6. Eje longitudinal Este-Oeste y ejes transversales. Reconstrucción del autor.

Una primera lectura del campo visual del complejo proporcionaría una percepción global relacionada con la visualización de distintas escenas. En este sentido, desde Vistas a un Mar Distante (Yuanying Guan), los visitantes podían contemplar sorpresivamente un impresionante paisaje, en cuya composición destacaba, además de la Gran Fuente (Dashuifa), y el Trono (Throne), una exuberante y cuidada vegetación, así como grandes $\operatorname{lagos}^{10}$ (mares), Figura 7. Según Jorgensen, “[...] para un arquitecto que diseña paisajes, existen tres elementos naturales: la forma del terreno, el agua y la vegetación" (Jorgensen, 1998: 44-45).

\footnotetext{
${ }^{10}$ Los lagos, fueron llamados mares en las dinastías del Norte y del Sur (420-581). Anteriormente, en la dinastía Han Occidental (206-224), el gran lago Kunming fue simbólicamente llamado mar, creándose una relación directa entre el agua estancada de un jardín y el mar.
} 


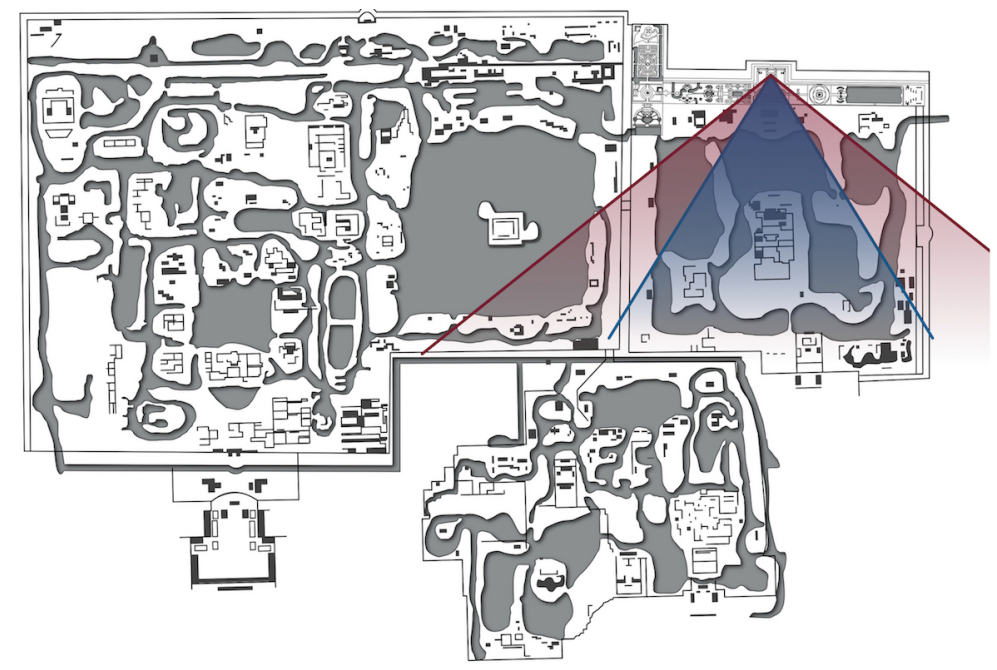

Figura 7. Campo visible desde el interior del Yuanying Guan. Reconstrucción e interpretación del autor.

Es significativo que el uso del término Guan como mirador o vista se limitara a tres palacios: Vistas más allá del Mundo (Belvedere o Bella Vista), Vistas a un Mar Distante (View of the Distant Sea o Yuanying Guan) y Visualización del Método de Agua (Throne). Teniendo en cuenta el gran número de recintos palaciegos del Jardín Europeo, y en general, del Yuanming Yuan, este signo de comunicación demuestra la importancia que tuvieron los Palacios Europeos en el entorno sintagmático de los Jardines. Esta primera reflexión semiótica sobre el Yuanying Guan, obliga a conocer el signo o propiedad global que identifica al palacio propiamente dicho como mirador. Entre otras interpretaciones que se pudieran dar, Guan significa literalmente mirar, visualizar, vista, y tiene múltiples connotaciones con la principal función del Palacio y Trono: mirador ${ }^{11}$ (Tabla 1. Indicador

\footnotetext{
${ }^{11}$ Durante la dinastía Western Han, el signo Guan estuvo relacionado con la idea de templo o edificio específicamente construido para visualizar lejanos paisajes. Su modo de signo
} 
C). Este signo guan connota con la visualización de escenas teatrales de paisajes ilusionistas. Para el emperador, el Yuanying Guan ${ }^{12}$ fue un refugio, un lugar para la contemplación escénica, una reducción microscópica de la naturaleza. Por estos motivos, el simbolismo está constantemente presente en él y los artistas jesuitas no fueron ajenos a ello.

Así mismo, la ubicación del Yuanying Guan en el centro axial ${ }^{13}$ (Tabla 1. Indicador D), del eje Este-Oeste del Jardín Europeo (Figura 2 y Figura 6), transmite un mensaje de centralidad, orden, geometría, jerarquía, influencia y dominación. Su construcción sobre una elevada plataforma fue concebida de forma que su eje coincidiera con el eje NorteSur del Jardín de la Eterna Primavera. Existió un rígido control geométrico en la disposición del palacio dentro del Jardín Europeo con el objetivo de conseguir una buena visualización de los lagos del Jardín de la Eterna Primavera y probablemente de otros lagos, como el Fukai Lake, ubicado en el Jardín de la Perfecta Brillantez. Esta centralidad constructiva connota un concepto de direccionalidad y añade un segundo nivel de articulación. Por otra parte, no fue casual la orientación Noreste del Jardín Europeo. Antiguos diagramas cosmológicos revelan que el signo gen $^{14}$ (Tabla 1.

como mirador, vistas, visualización, es indéxico porque tiene una relación causa-efecto con el significado. Su significado literal es denotado. A su vez es connotado porque nos induce a una interpretación subjetiva del significado. Connota con lagos distantes (mares), con embajadas extranjeras que llegaban a través de esos mares, con paisajes escénicos, teatralidad, etc. Metonímicamente representa la idea de mirador (Tabla 1. Indicador C).

${ }^{12}$ Guan fue un tipo de edificio construido específicamente para la visualización de lejanos paisajes durante la dinastía Western Han. Un templo daoista es usualmente llamado Guan. ${ }^{13}$ Es un signo cuyo significado está relacionado con centralidad, orden, geometría, jerarquía, influencia y dominación. Su modo es indéxico porque como puede verse en la fig. 6 está conectado con el significado produciendo un efecto central en el propio plano. Es icónico porque representa la idea de la centralidad. Su condición paradigmática viene avalada por ser una unidad del sintagma Palacios Europeos. Denota claramente con la posición geométrica del signo y connota con geometría, ejes, proporcionalidad. Tiene además doble articulación porque en el conjunto de unidades estructurales que construyen el centro axial se pueden establecer distintas relaciones de primer y segundo nivel (Tabla 1. Indicador D).

${ }^{14}$ Significante cuyo significado está relacionado cosmológicamente con el principio y fin de todas las cosas. Signo simbólico por ser puramente convencional y su dimensión 
Indicador E), significa dirección Noreste (NE). Una explicación sobre este signo ubicada temporalmente en la dinastía Qing dice: “Gen es el signo cosmológico del NE, dirección en la que todas las cosas empiezan y terminan. [...]. Beijing está localizada en el punto gen de China, lugar que puede percibir el retorno de todas las cosas. Está cerca del Norte, y recibe en la cara, la brillantez del Sur" (Minzhong, 2001: vol. 1, 82).

En este sentido, la ubicación del palacio en el Noreste mirando al Sur, basada en el principio confuciano, sentarse en el Norte con la cara al Sur, tiene además una dimensión simbólica cuya lectura semiótica explica una cierta protección mental para el disfrute del Emperador en aquel complicado laberinto de jardines. Esto explica que la planta del palacio (Figura 8) estuviera dividida en tres zonas, según la tradición china, siendo la zona central mayor que las dos laterales ${ }^{15}$ (Tabla 1. Indicador F). Estos dos cuerpos sobresalientes cumplían los códigos taoístas de la arquitectura china.

\footnotetext{
connotativa está basada en la diversidad de los distintos diagramas cosmológicos. Metonímicamente define una dirección (Tabla 1. Indicador E).

${ }^{15}$ Este significante tiene un significado de tradición, estabilidad y perfección. Su modo de signo es indéxico por su conexión con el significado constructivo de las zonas. Denota con la división literal planimétrica de la planta y connota con las tradiciones chinas respecto de la división horizontal del pabellón, geometría, direccionalidad Sur. Connota además con trono, iluminación, etc. Su articulación permite establecer relaciones (dimensiones, elementos, alusiones al Sur etc.) en la división tripartita (Tabla 1. Indicador F).
} 

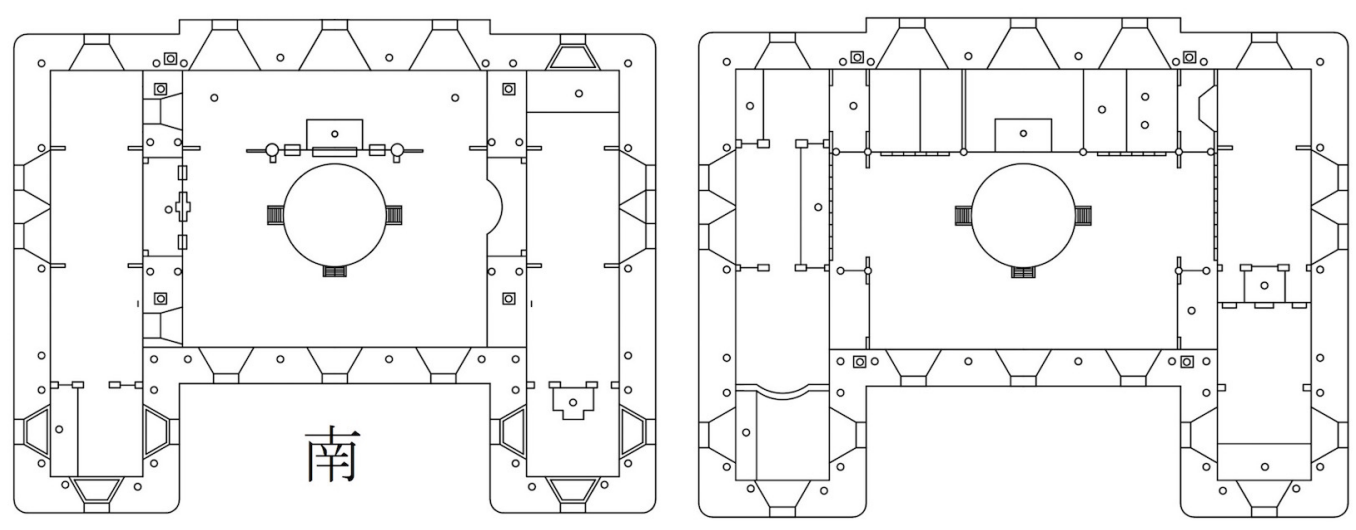

Figura 8. Reproducción de los planos del Yuanying Guan (Model Lei, Qing Dynasty). En el original, existe una inscripción lateral en chino cuya traducción es: "Yuanying Guan, plano. (Uno de los planos)", y en la parte frontal del plano de la izquierda, existe un carácter chino, 南, que significa Sur. Existen además anotaciones ilegibles en el interior de los planos, que se refieren a las medidas de los distintos elementos. Esta reproducción la ha realizado el autor a partir de los planos encontrados en (Zou, 2005: Figura 20).

Como se deduce de los planos de la Figura 8, el cuerpo del palacio tiene dos claros paradigmas: su división horizontal en tres zonas, y sus formas paralepipédicas, que transmiten mensajes de estabilidad $y$ perfección. Ambos paradigmas pueden ser incluidos en un sintagma que está codificado por las reglas y normas seguidas en la ejecución del Jardín Europeo: armonía, proporción y geometría y en las tipologías chinas para la distribución del espacio paisajístico.

En la zona central del plano de la Figura 8 existe un posible trono orientado al Sur y detrás una pantalla protectora ${ }^{16}$. Esta función de mirador ejercida hacia el Sur desde el trono interior del Palacio, a través de puertas y ventanas, posibilitaba la visión del Dashuifa (Gran Fuente), del Throne,

\footnotetext{
${ }^{16}$ Es significativo que en la parte frontal del plano exista un carácter chino nan, 南, que significa Sur, acentuando la importancia de esa orientación.
} 
de los grandes lagos distantes (mares), del Jardín de la Eterna Primavera y del Jardín de la Primavera Galante, aumentando así la sensación de poder del emperador. La Figura 9 muestra la situación geometrizada del trono interior del Yuanying Guan respecto del Dashuifa y el Throne:

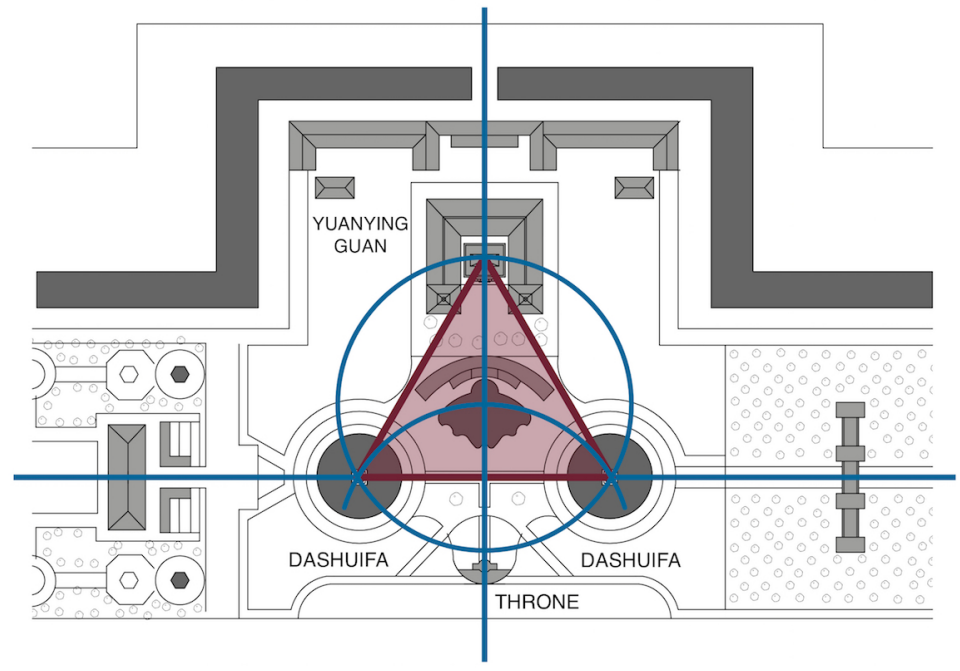

Figura 9. Geometrización del complejo del Yuanying Guan. Reproducción e interpretación del autor.

Abundando en reflexiones anteriores, una lectura adecuada de la expresión formal del palacio se identifica también con la función de mirador hacia el infinito. En la filosofía china, el punto del infinito está claramente identificado con el origen del mundo (Zou, 2005: 191). Así, Vistas a un Mar Distante, cuyo significado implica lejanía, connota a su vez con los mares lejanos (lagos), del Jardín de la Eterna Primavera, con la llegada de extranjeros a través de los mares, con paisajes escénicos etc. Montañas, ríos, mares, son percibidos como entes vivos y símbolos permanentes (Yi-fu, 1993: 128).

Teniendo en cuenta la base sobre la que se construyó el palacio (Figura 10 y Figura 11), es posible reconocer dos signos: en primer lugar, 
dicha base estuvo constituida por una plataforma elevada ${ }^{17}$ (Tabla 1. Indicador G) (terraza), y lógicamente tiene una connotación indexical con el signo guan o vistas. En segundo lugar, existe un gran número de ventanas a través de las cuales penetra la luz del Sur y podía contemplarse el vuelo sostenido del ave Fénix que debido a su naturaleza estaba relacionado con el Sur. En este sentido, la orientación que Castiglione dio a la fachada mirando al Sur, tenía el propósito de hacerla coincidir con la idea imperial de recibir la brillantez del Sur. En la tradición taoísta, la luz es la metáfora más usada para hacer referencia a la nuestra verdadera naturaleza, a la fuente de la vida. El uso de la luz del Sur como escudo protector, añade un segundo nivel de articulación en relación a la perfecta visibilidad de los escenarios. En este sentido se manifiestan los versos del poema Chanting at the View of the Distant Sea (Zou: 2011, 188):

Glass windows compose beautiful views.

Widely open windows on all sides lead to cool breezes.

On the emerald-green screen the purple phoenix lingers in fragrant mists.

A room with clear views is prepared for the distant sea.

\footnotetext{
${ }^{17}$ El significante elevación de la planta tiene un significado relacionado con la posición adecuada para servir de mirador. El modo del signo es indéxico porque está conectada al signo por una cierta causa-efecto y denota con el sentido literal de elevación. A su vez connota con distintas interpretaciones de la funcionalidad de la plataforma elevadora, mejor visibilidad y dominio, jardines, lagos, etc. (Tabla 1. Indicador G).
} 


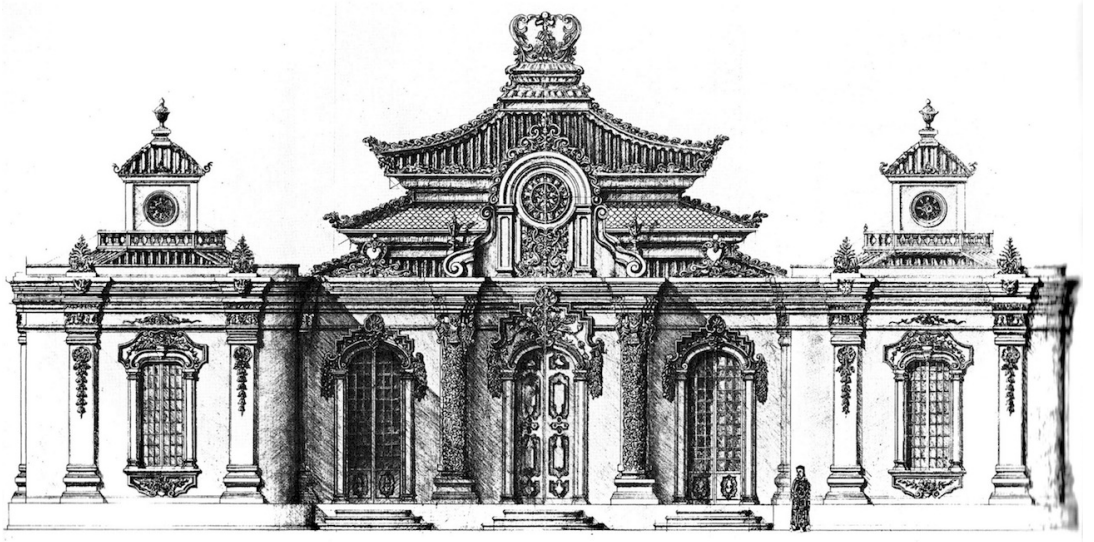

Figura 10. Fachada Sur del Yuanying Guan. Reconstrucción en papel por A. Durand (1985).

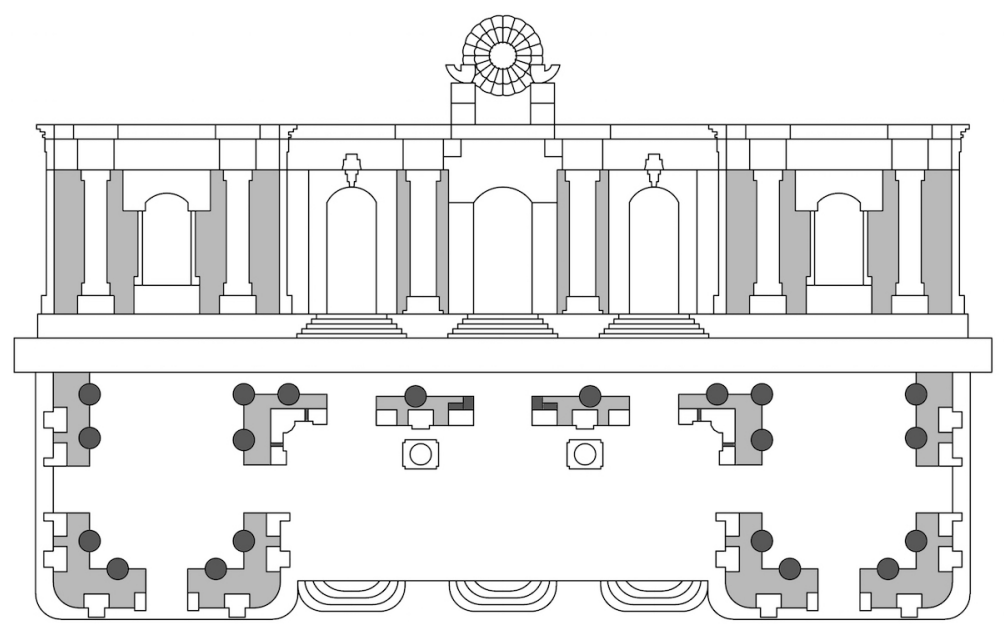

Figura 11. Reconstrucción de plano de la fachada Sur del Yuanying Guan.

Reproducción del autor.

En la zona delantera y alrededores del palacio, Castiglione jugó con el análisis de las formas, dando a la jardinería ornamental formas geométricas basadas en la simetría y la proporción (Arnheim, 1954). La verticalidad de algunos de sus elementos revela un mensaje de aspiración 
a Dios.

Una observación del plano de la fachada ${ }^{18}$ (Tabla 1. Indicador H), Figura 11, transmite un doble mensaje: ritmo y proporción, denotación de las clásicas villas italianas del Renacimiento Humanista. Ambos están basados en la original concepción griega del rhythmos o repetición de elementos a intervalos regulares, como indica J. Pollitt en su monografía The Ancient View of Greek (1974)

En un sentido más amplio, Vistas a un mar Distante, como paradigma asociado al sintagma Yuanming Yuan, es un elemento que transmite además un mensaje de geometrización del Jardín Europeo basado en el código xianfa o hibridación de la perspectiva lineal renacentista, con los métodos pictóricos chinos. El uso del xianfa, además de plasmar la realidad fundamentada en la perspectiva matemática lineal, también podría dibujar en la mente del espectador un paseo espiritual fundamentado en la idea taoísta ${ }^{19}$ (Tabla 1. Indicador I).

Existen además fuertes connotaciones del Yuanying Guan con la lírica literaria, la caligrafía, la pintura y la música. Su armonía y calculada simetría, connota con distintos detalles decorativos de estilo

\footnotetext{
${ }^{18}$ Este significante tiene un significado que está identificado con la repetición de elementos (ventanas, puertas, columnas etc), regularmente espaciados. El modo de signo indéxico utiliza dichos elementos para producir el efecto de proporcionalidad, iluminación y ornamentación. Así mismo, el simbolismo también está presente en el significante ya que la torre central y laterales revelan un mensaje de elevación. Denota con las fachadas de villas italianas renacentistas. La lectura connotativa debemos referirla a su relación con los códigos de la perspectiva matemática lineal, tradiciones, iluminación. Su disposición cara al Sur permite el sentido metafórico del signo. La articulación simple explica la posibilidad de relacionar sus unidades estructurales geométricamente y estéticamente (Tabla 1. Indicador H).

19El elevado número de ventanas tiene un significado indéxico debido al efecto de luminosidad que aporta y simbólico por la espiritualidad que implica la luz del Sur en la filosofía china. Su lectura semiótica explica una cierta protección mental para el disfrute del Emperador. El número de ventanas es un paradigma que representa las unidades que conforman gran parte del sintagma fachada. Denota con el efecto de luminosidad y connota con la ubicación geométrica, ornamentación y orientación que requiere la proporcionalidad de la fachada. Está además relacionado metafóricamente con la luz del Sur (Tabla 1. Indicador I).
} 
barroco. Jiaqing (reinó, 1796-1820), hijo de Qianlong, escribió un gran número de poemas dedicados a los pabellones del Jardín Europeo y más concretamente al Yuanying Guan. En uno de sus célebres poemas, titulado Writing on the View of the Distant Sea, el Emperador Jiaqing escribió: "The rooms imitate the Western style"/ "My little heart includes the distant seas"/ "The imperial mind embraces the great world" (Zou: 2011, 186).

Jiaqing hace una clara referencia a la influencia de Occidente y a pesar de considerarse "pequeño", su poder iluminado se agiganta hasta límites metafóricos cuando hace alusión a "abrazar la grandiosidad del mundo", representada en el microcosmo del Jardín Jesuita. Las dos escaleras existentes para el acceso a la Gran Fuente y Trono, construidas con material obtenido de rocas Taihu ${ }^{20}$, sublimaban la monumentalidad del Yuanying Guan $^{21}$ (Tabla 1. Indicador J). Por otro lado, los módulos paradigmáticos, Gran Fuente y Trono, transmiten distintos signos comunicativos que están relacionados con las fuentes mecánicas de tecnología occidental y su visualización desde el Trono. Así, la Gran Fuente, asociada a los Métodos de Agua de los Palacios Europeos, tuvo un antecedente en las fuentes mecánicas de relojería de la dinastía Ming, que provocaron gran curiosidad entre los Emperadores. El emperador Yongzheng, en su poema Chanting

\footnotetext{
${ }^{20}$ Las rocas Taihu son naturales y provenienen del lago Taihu. Tienen unas características (veteado y transparencia), que transmiten cierta sensación de misterio. Son muy apreciadas para su empleo en arquitectura.

${ }^{21}$ Yuanying Guan tiene una significación global de dominio sobre todo el complejo. Sus modos de signo indéxico y simbólico están relacionados con su significado como mirador. Su carácter paradigmático respecto del Jardín Europeo (sintagma), denota con la definición literal del palacio y connota con todos los códigos de la hibridación arquitectónica chino-europea desde los puntos de vista pictóricos y arquitectónicos. Su propio nombre, Vistas a un mar distante es una metáfora referida a contemplación de los grandes lagos del Jardín de la Eterna Primavera (ver fig. 6). La doble articulación hay que contemplarla desde su condición paradigmática. Para el nivel de primera articulación el palacio contiene pequeñas unidades de significación (ejemplo: en la ornamentación del palacio existen pequeñas unidades decorativas, como ventanas), cuyo código semiótico se divide en unidades funcionales mínimas que carecen de significados por sí mismas, pero que pertenecen al código (ejemplo: ventana se descompone en elementos, como marcos, cristales, etc. sin significación especial), o segundo nivel de articulación (Tabla 1. Indicador J).
} 
on an Automatic Clock, escribió: "The ingenious mechanism corresponds to heavenly rhythm"/ "The marks of time have no errors"/ "Clocks are extremely precise works", "Lotuses suspend in the mechanical clock" / "I doubt it is made by human labour" (Zou: 2005, 225).

En estos versos existen signos (p.e. el reloj mecánico), que denotan y connotan con la cosmología y su relación con la perfección de la tecnología occidental ${ }^{22}$ (Tabla 1. Indicador K). Así mismo, el lotus, originario de China y conocido antes de la llegada del budismo como la flor de la pureza y la integridad, aparece relacionado con la tecnología occidental. El lotus evoca además un estado de armonía (Siu, 1999: 389). El emperador pudo considerar que las vistas desde el Yuanying Guan constituían una escena pictórica de indudable belleza, que penetraba por sus ojos y la retenía en su mente. Las metáforas y metonimias abundan en los distintos poemas, así como elementos simbólicos de la cultura china. Todos los versos exaltan las bondades de la tecnología occidental a través del reloj al que el emperador relaciona con su conocimiento del cosmos.

El agua expresa inestabilidad emocional, inmediatez, contemplación, eternidad, añoranza (Cullen, 1961). En esta monumental obra se observa la gran originalidad de los artistas jesuitas para diseñar un proyecto de Norte a Sur y de Este a Oeste (Yufeng, 1984: 22).

Finalmente, como explican las fuentes consultadas en este trabajo, el Jardín Europeo, y más concretamente el complejo arquitectónico que hemos analizado, representa una poderosa imagen del imperio como un microcosmos. La mágica escenografía repleta de colorido, ornamentación y geometría constituye un fiel reflejo de los materiales utilizados en su ejecución, así como de su diseño chino-europeo. La gran cantidad de

\footnotetext{
${ }^{22}$ El significante, reloj de la fachada, fundamenta su significado en la tecnología occidental. El modo de signo es simbólico porque convencionalmente se adoptó esa representación para dicho significado. Es denotativo y connotativo con la mencionada tecnología y sus ramas, la cosmología y la armonía. Por último, es metafórico como se comprueba en algunos versos del emperador Yongzheng: Lotuses suspend in the mechanical clock or heavenly rhythm, p. 24 (Tabla 1. Indicador K).
} 
símbolos y signos que el Yuanying Guan muestra al investigador hacen de él un auténtico paradigma dentro del sintagmático Jardín Europeo. La presencia de significantes que pueden ser interpretados semióticamente, posibilita una apasionante reflexión sobre la lectura de su texto arquitectónico y refuerzan la idea de que todo el complejo estaba destinado a ser un mirador con innumerables modos de representación sígnica. Un conjunto de escenas materiales y sensoriales, donde el emperador era su actor principal.

\section{CONCLUSIÓN}

La arquitectura monumental transmite mensajes más allá de sus objetivos funcionales y contienen características expresivas y sensoriales relacionadas con los contextos históricos, religiosos, culturales y sociales del momento y lugar donde se desarrollaron. El análisis semiótico de los objetos arquitectónicos tiene como objetivo identificar las características del mensaje o del texto generado en dichos objetos para determinar el lenguaje visual que interrelaciona a todos sus elementos. La ayuda de la Semiótica es útil para comprender las tradiciones culturales que subyacen en un determinado objeto arquitectónico.

Aunque la literatura existente sobre la dimensión histórica del Jardín Yuanming Yuan es relativamente amplia, no existen análisis semióticos para una clara y concreta interpretación de sus significados. Sin embargo, existe la posibilidad de acercarnos a la idea de su conformación como un escenario no solo material sino también capaz de activar los sentidos. De ahí deriva su relación con la poesía, la música y la pintura. Su última ampliación, denominada Jardín Europeo implicó una difusión de la redonda brillantez, hacia el noreste, zona más lejana del Yuanmimg Yuan. Este hecho, en palabras de Qianlong: "encircle the cosmos and things into the round brightness", marcó claramente sus límites y supuso una forma de medir la perfecta brillantez de todo el jardín.

En este trabajo hemos utilizados conceptos y estructuras semióticas 
para interpretar el mensaje arquitectónico del Palacio Yuanying Guan creado a partir de una compleja interrelación de códigos y convenciones que la audiencia contemporánea desconoce. Los mensajes incorporados a los objetos arquitectónicos en forma de signos solo son comprendidos a través de dichos códigos, encargados de organizar los signos para que puedan ser leídos con la ayuda de herramientas semióticas.

Hemos comprobado, durante el desarrollo del trabajo, que el objeto arquitectónico analizado incorpora un texto simbólico que interpreta por una parte los deseos del emperador, sus aficiones y sus sentimientos, y por otra la intencionalidad apostólica de los artistas jesuitas.

El amplio campo de la hibridación arquitectónica y algunos conceptos e ideas taoístas se han utilizado como códigos suficientes en la mayor parte del trabajo desarrollado. Muchos de los signos analizados son connotativos y, desde un punto de vista denotativo, las funciones de algunos de sus elementos solo comunican y no tienen una función específica. En su conjunto, el palacio nos dibuja un lugar para el dominio escénico del Jardín Europeo. Hay que constatar que muchos de los signos utilizados en el Yuanying Guan son metafóricos, tales como los que se refieren a la lejanía, luz del Sur, la tecnología occidental, mares etc. y en algunos casos metonímicos. Respecto de las articulaciones aparecen algunos casos en nuestro análisis en los que no existe articulación, raramente aparece la doble articulación y es más frecuente la articulación simple.

Mediante el análisis desarrollado, ha quedado probado que el Yuanying Guan genera un lenguaje visual basado en la simbiosis de la cosmología china con la religiosidad jesuítica y la hibridación pictóricoarquitectónica chino-europea. Resumidamente, el objeto arquitectónico analizado se comporta como una representación teatral (signo), en la que el emperador fue el actor principal.

La Tabla 1 (para cada identificador A-K), resume la interpretación de los vocabularios y las reglas que gobiernan los aspectos semióticos más importantes del lenguaje visual del Yuanying Guan. Los conceptos y estructuras semióticas utilizadas ayudan a la comprensión de esta obra monumental. 


\begin{tabular}{|c|c|c|c|c|c|c|c|c|c|c|}
\hline \multirow{2}{*}{$\frac{\text { Indicador }}{\text { A }}$} & \multirow{2}{*}{$\begin{array}{l}\text { Significante } \\
\begin{array}{l}\text { GEOMETRIA DEL } \\
\text { JARDIN EUROPEO }\end{array}\end{array}$} & \multirow{2}{*}{$\begin{array}{c}\text { Significado } \\
\begin{array}{c}\text { REDONDEAR la } \\
\text { Perfecta Brillantez }\end{array}\end{array}$} & \multirow{2}{*}{$\frac{\text { Signo }}{\square}$} & \multicolumn{2}{|c|}{$\begin{array}{l}\text { Sintagma } \\
\text { Paradigma }\end{array}$} & \multicolumn{2}{|c|}{$\begin{array}{c}\text { Denotación } \\
\text { Connotación }\end{array}$} & \multicolumn{2}{|c|}{$\begin{array}{c}\text { Metáfora } \\
\text { Metonimia }\end{array}$} & \multirow{2}{*}{$\begin{array}{c}\text { Articulación } \\
\end{array}$} \\
\hline & & & & $\mathbf{S}$ & & D & C & ○ & & \\
\hline B & YUANYING GUAN & $\begin{array}{l}\text { MIRADOR HACIA EL } \\
\text { INFINITO }\end{array}$ & $\Delta$ & $\mathbf{S}$ & $\mathbf{P}$ & D & $\mathbf{C}$ & 0 & (0) & $\nLeftarrow *$ \\
\hline C & Término GUAN & $\begin{array}{l}\text { MIRADOR,VISTAS, } \\
\text { VISUALIZACION }\end{array}$ & $\boldsymbol{\Delta}$ & & & D & C & & ○ & \\
\hline D & $\begin{array}{l}\text { UBICACIÓN CENTRO- } \\
\text { AXIAL }\end{array}$ & $\begin{array}{c}\text { CENTRALIDAD, } \\
\text { ORDEN, } \\
\text { GEOMETRIA, } \\
\text { JERARQUA'A, } \\
\text { INFLUENCIA }\end{array}$ & $\frac{\Delta}{0}$ & & $\mathbf{P}$ & D & C & & & $\nLeftarrow$ \\
\hline E & $\begin{array}{l}\text { ORIENTACIÓN GEN } \\
\text { (NE) }\end{array}$ & $\begin{array}{l}\text { PRINCIIIO Y FIN DE } \\
\text { TODAS LAS COSAS }\end{array}$ & - & & & & C & & (ᄋ) & \\
\hline$F$ & $\begin{array}{l}\text { PLANIMETRIA: } \\
\text { DIVISION ENTRRS } \\
\text { ZONAS }\end{array}$ & $\begin{array}{l}\text { TRADICIÓN, } \\
\text { ESTABILIDAD, } \\
\text { PERFECCIONN }\end{array}$ & $\boldsymbol{\Delta}$ & & & D & C & & & $\nLeftarrow$ \\
\hline G & $\begin{array}{l}\text { ELEVACIÓN DE LA } \\
\text { PLANTA }\end{array}$ & $\begin{array}{c}\text { POSICIÓN } \\
\text { ADECUADA para } \\
\text { Servir de MIRADOR }\end{array}$ & $\Delta$ & & & D & C & & & \\
\hline $\mathrm{H}$ & $\begin{array}{l}\text { FACHADA YUANYING } \\
\text { GUAN }\end{array}$ & $\begin{array}{l}\text { RITMOY } \\
\text { PROPORCIÓN }\end{array}$ & $\Delta$ & & & D & C & 0 & & $\nLeftarrow$ \\
\hline I & $\begin{array}{l}\text { ELEVADO NÚMERO } \\
\text { DE VENTANAS }\end{array}$ & $\begin{array}{c}\text { Marco para } \\
\text { VISUALIZAR EL } \\
\text { PAISAEY LUZZ DEL } \\
\text { SUR }\end{array}$ & $\mathbf{\Delta}$ & & $\mathbf{P}$ & D & C & 0 & & \\
\hline J & MONUMENTALIDAD & $\begin{array}{l}\text { DOMINIO SOBRE EL } \\
\text { COMPLESO }\end{array}$ & $\Delta$ & & $\mathbf{P}$ & D & C & 0 & & $*$ \\
\hline K & RELOJ FACHADA & $\begin{array}{l}\text { TECNOLOG/A } \\
\text { OCCIDENTAL }\end{array}$ & a & & $\mathbf{P}$ & D & C & 0 & & \\
\hline
\end{tabular}
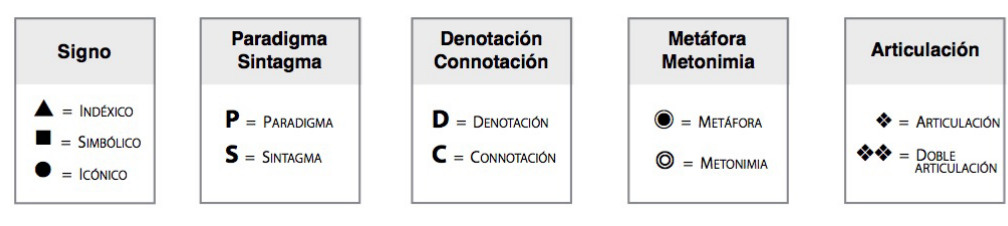

Tabla 1. Aspectos semióticos del lenguaje visual del Yuanying Guan. Realizada por el autor. 


\section{REFERENCIAS BIBLIOGRÁFICAS}

AGREST, D. \& GANDELSON, M. (1995). Semiotics and Architecture. New York: Princeton Architectural Press.

AMIRMASOUD, D. (2015). "The Reflection of Semiotic Theories in the Architectural Reading of the Contemporary Mosques of Tehran". Indian Journal of Fundamental and Applied Life Sciences 5.52, 2460-2471.

ARNHEIM, R. (1954). Art and Visual Perception. Berkeley: University of California Press.

BARMÉ G.R. (1996). East Asian History. Canberra: Division of Pacific and Asian History. Research School of Pacific and Asian Studies / Australian National University.

BARTHES, R. (1972). Le degré zéro de l'ecriture: Nouveaux essays critiques. Paris: Seuil.

BEURDELEY, C. \& BEURDELEY, M. (1971). Giuseppe Castiglione: A Jesuit Painter at the Court of the Chinese Emperors. Traducción de Michel Bullock. Rutland, Vt. and Tokyo: Charles E. Tuttle Co. BEURDELEY, M. (1997). Peintres jésuites en Chine au XVIII siècle. Arcueil Cedex: Anthèse.

BOTTON, F. (2005). "Reseña del libro de Elisabetta Corsi, La fábrica de las ilusiones. Los jesuitas y la difusión de la perspectiva lineal en China (1698-1766)". Estudios de Asia y África 40.2 (127), 465467.

BROADBENT, G. (1977). “A Plain Man's Guide to the Theory of Signs in Architecture”. Architectural Design 7-8, 474-482.

CASTILLA, M. V. (2015). "The Apostolic Status of Linear Perspective during the Eighteenth-Century in China". Revista Orientando (Centro de Estudios China-Veracruz) 10 (abril-septiembre), 1-24. (2016). "Giuseppe Castiglione (Lang Shining). Precursor de la primera mundialización pictórico-arquitectónica”. Estudios de Asia y África (El Colegio de México) 161 (septiembre), 623-646. 
CORSI, E. (2004). La fábrica de las ilusiones. Los jesuitas y la difusión de la perspectiva lineal en China (1698-1766). México: Centro de Estudios de Asia y África (El Colegio de México).

CUllEN, G. (1961). The Concise Townscape. London: Architectural Press.

CHANDLER, D. (2007). Semiotics: The Basics. Second Edition. London: Routledge.

DONOUGHO, M. (1987). "The Language of Architecture". The Journal of Aesthetic Education 21.3, 53-67.

ECO, U. (1986). La estructura ausente. Introducción a la Semiótica. Barcelona: Editorial Lumen.

(1997). "Function and Sign: The Semiotics of Architecture". En Rethinking Architecture: A Reader in Cultural Theory, Neil Leach (ed.), 173-195. London: Routledge.

FISKE, J. (1990). Introduction to Communications Studies [2 ${ }^{\text {nd }}$ Edition]. London: Routledge.

GREIMAS, A. J. \& COURTÉS, J. (1982). Semiotics and language: An Analytical Dictionary. Bloomington: Indiana University Press.

HALE, J.A. (2000). Building ideas: An Introduction to Architectural Theory. Chichester: John Wiley \& Sons LTD.

JENCKS, Ch. \& BAIRD, G. (eds.) (1969). Meaning in Architecture. New York: George Braziller.

JENKCS, Ch. (1984). "El signo arquitectónico". En El lenguaje de la arquitectura: un análisis semiótico, G. Broadbent, R. Bunt \& C. Jenkcs (eds.), 79-127. México: Limusa.

JORGENSEN, K. (1998). "Semiotics in Landscape Design". Landscape Review 4.1, 39-47.

JUODINYTÉ-KUZNETSOVA, K. (2011). “Architectural space and Greimassian semiotic". Societal Studies 3.4, 1269-1280.

KLEUTGHEN, K. (2013). "Staging Europe: Theatricality and Painting at the Chinese Imperial Court". Studies in Eighteenth-Century Culture 42, 81-102. 
LOEHR, G. R. (1940). Giuseppe Castiglione (1688-1766). Pittore di corte di Ch'ien Lung, Imperatore della Cina. Roma: Instituto Italiano per il Medio ed Estremo Oriente.

MAGARIÑOS DE MORENTÍN, J. (2007). "La Semiótica de los bordes. Significación y negatividad”. Tópicos del Seminario 18 (juliodiciembre), 99-100.

MARTIN, B. \& RINGHAM, F. (2000). Dictionary of Semiotics. London and New York: Cassell.

MINZHONG, Y. (2001). Ed. Rixia juwen kao. 4 vols. (Beijing: Beijing guji chubansh), vol.1, 82. [Cita tomada de la tesis de doctorado de Hui Zou, The Jing of Line-Method: A Perspective Garden in the Garden of Round Brightness. Montréal: School of Architecture, McGill University, 2005.]

NÖTH, Winfried (1990). Handbook of Semiotics. Bloomington: Indiana University Press.

PANIAGUA, E. (2013). “La Arquitectura y su significación pragmática y tectónica”. Signa 22, 521-548 (también en https://doi.org/10.5944/ signa.vol22.2013.6365 [12/04/2018]).

PEIRCE, Ch. S. (1931-1958). Collected Writings. 8 vols. Charles Hartshorne, Paul Weiss \& Arture W. Burks (eds.). Cambridge, MA: Harvard University Press.

PIRAZZOLI-T'SERSTEVENS, M. (2007). Giuseppe Caastiglione.

1688-1766, Peintre et architecte à la cour de Chine. Paris: Thalia édition.

POLLITT, J.J. (1974). The Ancient View of Greek. New Haven: Yale University Press.

POZZO, Andrea (1693 [1989]). Perspective in Architecture and Painting. Copia íntegra en latín e inglés de la edición de 1693, Perspectiva Pictorum et Arquitectorum. New York: Dover Publications, Inc., 1989.

RAWSKI, E. \& RAWSON, J. (2006). China: The three Emperors 16621795. London: Royal Academy of Arts. 
ROSSI, A. (1982). The Architecture of the City. [Primera edición. 1966]. The Graham Foundation for Advanced Studies in the Fine Arts, and The Institute for Architecture and Urban Studies. Cambridge: MIT Press.

SAUSSURE, F. (1983). Course in General Linguistics. London: Duckworth.

SIU, V. M. (2012). "China and Europe entwined: a new view of the European sector of the Chan Chun Yuan". Studies in the History of Gardens \& Designed Landscape 19.3-4, 376-393.

STANDAERT, N. (2007). An Illustrated Life of Christ Presented to the Chinese Emperor: The History of Jincheng Shuxiang (1640). Monumenta Serica Monograph Series, vol. LIX. Sankt Augustin: Steyler Verlarg.

TASHEVA, S. (2012). Semiotics of Architectural Graphics. Sofia: Institute of Art Studies.

TAURENS, J. (2008). "Meaning and Context in the Language of Architecture". En Place and Localization: Studies in Environmental Aesthetics and Semiotics VI, 71-82 Tallinn: The Research Group of Cultural and Literary Theory, Estonian Literary Museum, Institute of Art History, Estonian Academy of Arts. Estonian Semiotics Association.

THIRIEZ, R. (1994). “The Qing Long Emperor's European Palaces”. En The Delights of Harmony: The European Palaces of the Yuanming Yuan \& the Jesuits at the Eighteenth-Century Court of Beijing, 6-17. Worcester: Iris and B. Gerald Cantor Art Gallery, College of the Holy Cross.

WALEY-COHEN, J. (1999). The Sextants of Beijing: Global Currents in Chinese History. New York: W.W. Norton \& Company.

WONG, Y. (2001). A Paradise Lost: The Imperial Garden Yuanming Yuan. Honolulu: University of Hawai's Press.

YI-FU, T. (1993). Passing Strange and Wonderful: Aesthetics, Nature and Culture. New York: Island Press. 
YUFENG, J. (1984). Yuanming Yuan Lou Pinxi (A critical analysis of the European section in the Yuanming Yuan). Yuanming Yuan Garden Journals (YMYJ). Vol. 3, 21-24.

ZOU, H. (2005). The Jing of Line-Method: A perspective Garden in the Garden of Round Brightness. PhD. School of Architecture. Montreal: McGill University Montreal.

(2011). A Jesuit garden in Beijing and Early Modern Chinese Culture. West Lafayette: Purdue University Press, Project Muse.

Recibido el 11 de enero de 2018.

Aceptado el 11 de abril de 2018. 